University of Wollongong

Research Online

Australian Institute for Innovative Materials -

Papers

Australian Institute for Innovative Materials

$1-1-2015$

Probe current determination in analytical TEM/STEM and its application to the characterization of large area EDS detectors

David R. G Mitchell

University of Wollongong, dmitchel@uow.edu.au

Mltchell John Bromley Nancarrow

University of Wollongong, nancarro@uow.edu.au

Follow this and additional works at: https://ro.uow.edu.au/aiimpapers

Part of the Engineering Commons, and the Physical Sciences and Mathematics Commons

Research Online is the open access institutional repository for the University of Wollongong. For further information contact the UOW Library: research-pubs@uow.edu.au 


\title{
Probe current determination in analytical TEM/STEM and its application to the characterization of large area EDS detectors
}

\author{
Abstract \\ A simple procedure, which enables accurate measurement of transmission electron microscopy \\ (TEM)/STEM probe currents using an energy loss spectrometer drift tube is described. The currents \\ obtained are compared with those measured on the fluorescent screen to enable the losses due to \\ secondary and backscattered electrons to be determined. The current values obtained from the drift tube \\ allow the correction of fluorescent screen current densities to yield true current. They also enable CCD \\ conversion efficiencies to be obtained, which in turn allows images to be calibrated in terms of electron \\ fluence. Using probes of known current in conjunction with a NiO reference specimen enables the X-ray \\ detector solid angle to be determined. The $\mathrm{NiO}$ specimen also allows a wide range of other EDS detector \\ parameters to be obtained, including the presence of ice and carbon contamination. A range of \\ performance characteristics are reported for two large area EDS detector systems. Many of the \\ measurements reported herein have been automated via the use of freely available scripts for \\ DigitalMicrograph.
}

\section{Keywords}

area, large, characterization, application, its, detectors, stem, eds, tem, analytical, determination, current, probe

\section{Disciplines}

Engineering | Physical Sciences and Mathematics

\section{Publication Details}

Mitchell, D. R. G. \& Nancarrow, M. J. B. (2015). Probe current determination in analytical TEM/STEM and its application to the characterization of large area EDS detectors. Microscopy Research and Technique, 78 (10), 886-893. 
Probe current determination in Analytical TEM/STEM and its application to the characterisation of large area EDS detectors.

David R. G. Mitchell ${ }^{+}$and Mitchell J. B. Nancarrow

Electron Microscopy Centre

Innovation Campus

University of Wollongong

Squires Way, North Wollongong

NSW 2500, Australia

+ corresponding author: dmitchel@uow.edu.au, tel + 61242215312

Running title: Probe current determination in analytical TEM/STEM.

Key words: beam current measurement, transmission electron microscopy, $\mathrm{x}$-ray microanalysis 


\section{Abstract}

A simple procedure which enables accurate measurement of TEM/STEM probe currents using an energy loss spectrometer drift tube is described. The currents obtained are compared with those measured on the fluorescent screen to enable the losses due to secondary and backscattered electrons to be determined. The current values obtained from the drift tube allow the correction of fluorescent screen current densities to yield true current. They also enable CCD conversion efficiencies to be obtained, which in turn allows images to be calibrated in terms of electron fluence. Using probes of known current in conjunction with a $\mathrm{NiO}$ reference specimen enables the $\mathrm{x}$-ray detector solid angle to be determined. The $\mathrm{NiO}$ specimen also allows a wide range of other EDS detector parameters to be obtained, including the presence of ice and carbon contamination. A range of performance characteristics are reported for two large area EDS detector systems. Many of the measurements reported herein have been automated via the use of freely available scripts for DigitalMicrograph. 
Introduction

For quantitative analysis using standards in the scanning electron microscope or electron microprobe, a good understanding of beam current is essential (Goldstein and others, 2003). For this reason, such instruments are often fitted with an insertable Faraday cup and a dedicated picoammeter to enable current measurement. The Faraday cup is used in preference to a grounded fluorescent screen, since it captures incoming electrons, as well as secondary and backscattered electrons arising from the energetic interactions of the primary beam with the target (Williams and Carter, 2009). Since standards-based quantitative analysis with transmission electron microscopy (TEM) is generally not possible, due to the difficulty in controlling or knowing the thickness of the thin foil, TEMs typically do not have an insertable Faraday cup. Some vendors sell TEM holders with a Faraday cup built into them (Gatan, 2015). Electron energy loss spectrometers (EELS) fitted with drift tubes make an efficient Faraday cup, and the latest generation of Gatan Imaging Filters (GIF) have a picoammeter built-in with which to make current measurement straightforward.

Most TEMs will provide a read out of current (or current density) via the fluorescent screen. In order to convert current density to current, the area of the screen is required. This information is generally not well known. A further complication exists for the small insertable fluorescent screen. Historically, this has been used to set the exposure for the film camera, and the area used for current density purposes is not the geometric area, but rather the projection onto the film plane in the film camera. For this reason, a measured area for the screen may not enable accurate conversion of current density into current. Even then, backscattered and secondary electron losses are not included and so the reported current densities may be in error by 20-50\% (Williams and Carter, 2009). Microscopes from some vendors include a TV camera for viewing the TEM image. However, a fluorescent screen is still part of the imaging chain. Where such cameras report current directly, it is still desirable to confirm that the values are accurate.

Experienced electron microscopists will have a good feel for probe/condenser aperture conditions which are appropriate for various forms of imaging and analysis. However, reporting analytical conditions in terms of spot and aperture size makes such conditions not readily transferrable between instruments - even if from the same vendor. It is therefore desirable to know the probe currents used in various forms of imaging and analysis. Such information enables images to be calibrated in terms of electrons per unit area - often the preferred unit for cryo-EM and low dose imaging (Dubochet and others, 1988; Sousa and Leapman, 2007). Accurate current determination enables consistent in-situ electron beam processing and modification of materials to be carried out (Mackovic and others, 2014) and quantitative STEM measurement of molecular masses (Sousa and Leapman, 2007). Working with real currents also enables a detailed understanding of $\mathrm{x}$-ray detector response, especially where the solid angle is being measured (Egerton and Cheng, 1994)

This article describes some practical experimental procedures used for the measurement of probe current on analytical TEM/STEMs. No Faraday cup (or a holder with one fitted) was available on either instrument. The instruments were fitted with Gatan Imaging Filters (GIF). Neither imaging filter had the built-in picoammeter option. The method employed relies on using the Drift Tube of the GIF as a Faraday cup for measuring the probe current directly. Such measurements require only minor configurational changes to the GIF. However, as a safety precaution, the GIF is not operable when measuring current, so the method cannot be used for continuous online measurement. Since 
the drift tube current is reasonably accurate, it enables the relationship between fluorescent screen current density and the actual current to be obtained and the backscatter/secondary electron correction factor to be measured accurately. This relationship enables online current measurements to be performed effortlessly using the fluorescent screen/TV camera. By exposing the charge coupled device (CCD) camera(s) to probes of known current, the conversion efficiency of the CCD (counts per electron) can also be obtained. This enables images to be calibrated in electrons or electrons per unit area. Accurately derived probe currents have been used to determine the solid angle of a newly installed large area EDS detector. Some other detector parameters of interest are also investigated, including the extent of ice and carbon build-up on a windowless EDS detector array.

Materials and Methods

Two microscopes were used in this study:

FEI Osiris, fitted with a ChemiSTEM ( 4 x silicon drift detector -0.9 sr solid angle), Gatan Ultrascan top mounted camera and a Gatan Tridiem Imaging Filter (GIF).

JEOL ARM 200F fitted with a top-mounted Gatan Ultrascan camera, a Gatan Quantum Imaging Filter with Ultrascan camera and a JEOL Large Area EDS detector (1sr solid angle).

Measurements with the Osiris were carried out at 200kV while on the ARM they were performed at 80 and 200kV. Probe current measurements were made using a high impedance Digital Multimeter (DMM) and a Keithley 6485 Digital Picoammeter. EDS detector characterisation was performed using the procedures recommended for the NiOx reference specimen from Ted Pella Inc. (NiOx EDS reference specimen, 2015).

Current measurements were made using the GIF Drift Tube. As this can have very high voltages applied to it during normal operation it was essential that the Drift Tube voltage (set in DigitalMicrograph/Filter Control) was set to zero and the GIF power supply (GIB) was powered down prior to disconnecting cables. With the GIF powered down, the magnetic prism was not active and electrons entering the spectrometer would impact on the drift tube wall, and register as a current. The outer shield of the Drift Tube cable is connected to the microscope ground via the GIF. However, as a precaution against a faulty or poor earth, a grounding point on the microscope column itself was located and connected to the outer shield on the Drift Tube supply cable. The reliability of this earth connection was tested with a continuity meter. As the drift tube is electrically isolated when this supply cable is detached from the supply electronics it has the ability to float to a high potential when exposed to the electron beam. A continuous and reliable path to ground (via the current measurement device) is therefore essential. To ensure reliable connections, a break-out box was built which allowed the safe high voltage (SHV) connector on the drift tube cable to be patched through to a standard BNC-type connection on the picoammeter. Connection posts for a Digital Multimeter (DMM) connection were also included in the break-out box.

Analytical TEM/STEM probe currents are typically in the $\mathrm{PA}$ - nA range. A dedicated picoammeter is ideal tool for such measurements. However, where such an instrument is not available, an inexpensive alternative is a DMM. The majority of measurements reported here were made with a 
DMM as initially, a dedicated picoammeter was not available. They were subsequently validated against a Keithley digital picoammeter and were found to agree to within $1 \%$. Most DMM's minimum current measurement ranges $(\mu \mathrm{A})$ are too high for direct measurement of probe currents ( $n A-p A)$. Instead, the millivolt range was selected on the DMM and the voltage output from the drift tube measured. Modern DMMs have a very high internal resistance. The voltage measured is the potential developed across this internal resistance, which from Ohm's Law allows the current to be derived. The internal resistance of the DMM can be determined by measuring it with a second DMM. Higher internal resistance values $(\geq 10 \mathrm{~m} \Omega$ ) are better, since for a given probe current a larger voltage will be registered. Inexpensive DMMs may have resistances of just a few $M \Omega$ and this makes them too insensitive for this type of measurement.

Probe currents were varied by choosing a range of different spots sizes and condenser apertures in TEM and STEM mode. Screen currents were measured by converging the beam so that the beam was about $80 \%$ of the diameter of the fluorescent screen (large or small insertable) on which measurements were performed. In STEM mode all currents were measured on the small insertable screen and camera length was used to control the footprint of the beam. In the case of the FEI Osiris, which uses a TV camera (to capture images from an insertable fluorescent screen), the beam was converged to be fully contained with the frame of the TV image. Highly converged beams were not used during current measurement on fluorescent screens, as this can magnify inhomogeneities in the screen's response. For example, converging the beam on the small black focusing dot at the centre of the insertable screen resulted in a ca $10 \%$ higher net screen current compared with a beam spread to $80 \%$ of the screen diameter. This may reflect a lower backscatter/secondary electron yield from the black dot region, compared with the screen as a whole. The scintillator is most likely zinc sulphide or similar, whereas the black dot material may be a carbon-rich paint with a much lower mean atomic number. Secondary and backscattered yields from a material decrease with decreasing mean atomic number (Trump and Van De Graaff, 1947).

Drift tube currents were measured by converging the beam to the smallest size possible and raising the fluorescent screen to allow the beam to enter the GIF. The largest GIF entrance aperture was used to make beam alignment easier. Due to the capacitance of the drift tube, voltages were allowed to stabilise for 30s prior to measurement. Beam/Projector shifts were adjusted to ensure that the converged beam was correctly centred in the GIF entrance aperture.

Since the GIF electronics were powered down during current measurements for safety, it was not possible to measure a probe current and the GIF CCD conversion efficiency at the same time. The first stage of the process was to determine the relationship between current density measured on the fluorescent screen and current measured with the drift tube. Subsequently, the fluorescent screen was used to measure the current density and thus current, and the CCD camera was then exposed to such probes. Before measurement, camera dark references were removed and the camera temperature kept at steady state temperature overnight. Binning was set to $1 x$ and the beam was spread to fill as much of the frame area as possible, without running off the edge. The Ultrascan cameras have a saturation level of ca 65,000 counts, and exposure times were varied to capture around 40,000 cts at $1 x$ binning. 
Results and Discussion

FEI Osiris Current Calibration

Unlike the ARM microscope which uses a conventional imaging fluorescent screen, the Osiris uses a TV camera to display images (formed on an insertable fluorescent screen). The TV camera interface (FluCam) reports current directly, rather than current density in the case of JEOL ARM200F. It was desirable to check the accuracy of this current readout. Fig. 1 shows the plot of current measured at the drift tube versus that indicated on the Camera Interface. The slope is very close to unity (0.992) and the $R^{2}$ value is one. This result suggests that the current readout is indeed accurate and that the measurement method used here is robust.

\section{JEOL ARM Current Calibration}

JEOL microscopes display fluorescent screen current as a current density in pA. $\mathrm{cm}^{2}$. To convert this current density into current, the screen area is required. Since this is not reported in the JEOL documentation it must be measured. This is most easily achieved by photographing the fluorescent screen through the viewing window. The diameter of the screen can then be measured using the graduated scale etched on the fluorescent screen as an internal calibration. The screen area was found to be $269 \mathrm{~cm}^{2}$. This screen area enabled current density to be converted into current. A plot of drift tube current vs fluorescent screen current for measurements at 80 and $200 \mathrm{kV}$ resulted in straight line plots (Fig. 2). However, the slopes were not unity reflecting the fact that the Drift Tube collects all current while the fluorescent screen loses some to backscatter and secondary electron emission. In order to bring the drift tube and fluorescent screen currents into registry the latter must be multiplied by the relevant correction factor (Table 1). The higher values at $80 \mathrm{kV}$ are not unexpected since the backscatter and secondary electron yield increase as the accelerating voltage is decreased (Trump and Van De Graaff, 1947).

JEOL Fluorescent screen/current relationships.

From an operational perspective, drift tube current measurements take some time to set up and preclude the use of the GIF during measurement. A far more facile method of current measurement is to use the current densities reported by the various fluorescent screens and convert them to current directly. The approach discussed above enables both the BSE/SE correction factor and the geometric area of the fluorescent screens to be determined accurately. The screen geometric area is constant and the BSE/SE yield reasonably so, although with use, the screen will age and will become coated with cracked hydrocarbons. Its secondary and backscattered electron emission characteristic may therefore change with time.

Applying the direct measurement method to the small fluorescent screen is problematic as the screen is small, inclined to the beam and it is the area projected onto the film plane which is required. To avoid these complications, an alternative approach exists in which the relationship between screen current density and (drift tube measured) current is obtained. Here neither the screen area nor the BSE/SE correction factor are considered. The linear relationship between the 
current and current density enables direct conversion of one into the other. Fig. 3 shows the relationship obtained from measurements at $200 \mathrm{kV}$ using the small and large screen. The ratio of slopes in the two plots being the ratio of the respective screen areas.

The plots in Fig. 3 should (ideally) go through the origin. However, this is not the case. For measurements using the large screen, the intercept with the drift tube current axis occurs at $25.2 \mathrm{pA}$. Clearly, such a relationship cannot be used to estimate low currents. For a given probe current the large screen will report a much lower screen current density than the small screen - by a factor of 29.5x. Therefore, the large screen lacks the sensitivity to measure low current probes accurately, and the small fluorescent screen is preferred. In practice, the small screen should be used for all current measurements, up to the point where the probe current saturates the small screen's dynamic range $\left(512 \mathrm{pA} . \mathrm{cm}^{2}\right)$. This occurs at a current of approximately $5 \mathrm{nA}$. For currents greater than this, the large screen would be used. To expedite the conversion of screen current densities a convenient conversion script running in DigitalMicrograph has been created (Current Converter (Mitchell, 2014b)). This takes as inputs the microscope voltage and either the large or small screen current density. The probe current is then returned. The straight line fit constants derived from Fig. 3 are readily edited within the script, to enable users to modify them to suit their system.

\section{CCD Conversion Efficiencies}

High end charged coupled device (CCD) cameras used in electron microscopy such as the Gatan Ultrascan, rely on a scintillator to convert high energy electrons into light. This scintillator is then coupled to the camera via a fibre optic bundle. Electrons arrive at the scintillator with a specific quantum of energy, and via the production of light an output signal is generated from the camera. A parameter of interest is the CCD conversion efficiency which defines the efficiency of this process. It is reported in counts per electron and values may vary widely depending on beam energy, scintillator type and camera model and even within identical camera systems. The conversion efficiency is invariably reported in the certification supplied with the camera - but usually only at a single accelerating voltage, being the upper voltage at which the device is to be operated. It is desirable to calibrate the camera response to validate this value and more importantly to determine the conversion efficiency at lower voltages - since the values will change with voltage quite significantly. The advantage of calibrating the camera in terms of equivalent electrons is that it enables the local electron fluence the specimen received during imaging to be computed. This may be described in terms of numbers of electrons per pixel or as electrons per unit area. Such values are routinely used in low dose microscopy such as cryo-em (Dubochet and others, 1988) and single particle analysis (Bai and others, 2013), but are likely to be of interest to materials microscopists who are studying beam-sensitive materials/radiation damage effects (Egerton and others, 2004; Jiang and Spence, 2012; Sader and others, 2010).

Cameras were exposed to a range of different probes currents using exposure times of between 0.5 and $4 \mathrm{~s}$. The total counts in the images were summed. From the known beam current and time, the corresponding electron fluence was obtained. Table 2 shows the conversion efficiencies of the two Ultrascan cameras fitted to the ARM. The upper camera's (Gatan Ultrascan 2k x 2k, Model 994J77P2, scintillator $\mathrm{P}+$ ) quoted conversion efficiency at $200 \mathrm{kV}$ was 4.0 and the value measured here was 4.8 . 
The GIF mounted Camera (Gatan Ultrascan 2k x 2k, Model 963.J773PTNS, scintillator P) had a quoted conversion efficiency of 6.9 at $300 \mathrm{kV}$. Since the conversion efficiency will increase with decreasing voltage, the value measured here at $200 \mathrm{kV}$ of 8.0 seems reasonable. The reason for the significant difference between the two cameras is that the GIF-mounted camera has a different type of scintillator (type P compared with $\mathrm{P}+$ ). The higher efficiency of the GIF mounted camera offers advantages in energy filtered TEM mapping, where signal intensities can be extremely low, albeit at the expense of dynamic range. The measured conversion efficiencies at $80 \mathrm{kV}$ were approximately double those at 200kV (Table 2). For yttrium aluminium garnet (YAG) type scintillators used here, the peak in output per input electron has been reported to occur in the 100-120kV range with output falling at higher voltages (Zuo, 2000).

At GIF installation, the CCD conversion efficiency is usually stored within the tag structure of DigitalMicrograph to enable the intensity calibration to be applied automatically. However, this is generally not present for non-GIF-based cameras. To enable such calibrations to be applied in a facile manner, a freely available dialog-based calibration tool has been developed for DigitalMicgraph (CCD Electron Calibrator (Mitchell, 2014a)). This enables the calibration factors in Table 2 to be applied to images after acquisition. The tool has the option to include data from up to four different cameras and at four different voltages. Calibration can be performed in electrons, electrons per $\AA^{2}$ or electrons per $\mathrm{nm}^{2}$. Values may be applied to open images or to folders of saved images, enabling rapid calibration.

\section{EDS Detector Characterisation.}

The characterisation of an EDS detector immediately following installation or service can provide useful benchmarks to ensure satisfactory performance. It also provides a benchmark against which any possible deterioration in performance during service can be assessed. Evaporated $\mathrm{NiO}$ thin films have been suggested as being a very useful material for this evaluation (Egerton and Cheng, 1994). These are readily available from a commercial supplier, branded as 'NiOx' (NiOx EDS reference specimen, 2015). This specimen was used to determine various EDS detector characteristics of a JEOL large area (1sr) EDS detector on the ARM and the ChemiSTEM 4 x SDD detector array (0.9sr) on the FEI Osiris.

From a knowledge of the probe current, composition and thickness of an NiO film, it is possible to compute the x-ray flux generated. The ratio of the detected $x$-ray flux to the total emitted $x$-ray flux enables the solid angle of the detector to be derived. There are some uncertainties with this measurement, most notably the potential for the film not being stoichiometric $\mathrm{NiO}$ and/or deviating from the expected density. Amorphous Ge thin films have been suggested as a superior alternative to $\mathrm{NiO}$ (Zaluzec, 2014), but unfortunately such films are not yet available commercially.

JEOL Detector - Effect of tilt.

Specimen tilt is one of the many parameters which can influence the $x$-ray count rate and thus the effective solid angle. To assess the influence of specimen tilt, the NiO specimen was tilted between $+/-30$ degrees in $X$ (positive tilts are towards the detector of the ARM). Fig. 4 shows the tilt 
sensitivity. A $1 / \cos$ (tilt) correction has been applied to account for the increase in projected thickness with tilting. The $x$-ray count rate increases up to a tilt of +12 degrees (tilt about holder axis) and plateaus thereafter. This response is a consequence of the part of the detector being shadowed by the TEM holder and/or specimen at lower tilts. All JEOL detector performance measurements were therefore made at +14 degrees tilt. The $x$-ray count rate decreased with increasing negative tilt, and reached a minimum at approximately -16 degrees, before increasing again. Since the detector is so large $\left(100 \mathrm{~mm}^{2}\right)$ it is able to detect $x$-rays from both sides of the specimen within this tilt range. This is very beneficial in that at no point is the $x$-ray signal lost, as is invariably the case with smaller detectors. Even at its lowest yield position, the detector still generates a count rate approximately one third that of maximum. For microanalysis of cross-sections of interfaces, tilting to the edge-on condition is essential and invariably this tilt position is not towards the detector. The large area detector precludes the need to reposition the specimen within the holder.

\section{JEOL Detector - Effect of Specimen Orientation}

The orientation of the film on the grid is also another factor which affects $x$-ray yield. Measurements were made with the $\mathrm{NiO}$ film on the upper surface of the grid and with the specimen inverted. The film uppermost orientation helps minimise shadowing of the detector by grid bars/holder and therefore increases the solid angle. However, with the film uppermost scattered electrons are able to excite emission from the grid bars more readily, resulting in a decreased peak to background ratio. To assess this effect, spectra from the NiOx film were obtained in both orientations. The NiOx specimen enables a range of detector parameters to be obtained, including: solid angle, Fiori number, peak to background ratio and detector resolution. To aid computation of these data a convenient and freely available DigitalMicrograph script has been created (SuperX Monitor (Mitchell, 2014c)). This takes as its input EDS spectra obtained from the NiOx specimen. A wide range of spectrum formats can be read in from several different EDS systems. The only user-supplied data required are the accelerating voltage, the beam current, the film thickness and the acquisition time (live time). Peak fitting can be adjusted interactively to deal with any system peaks, peak shifts due to calibration errors etc.

Table 3 shows the effect of having the NiO film on the upper or lower side of the specimen during analysis. The probe currents $(0.356 \mathrm{nA})$, pulse processor times (slowest) and specimen tilt (+14degs) were identical for both series of measurements (average of four measurements per orientation). The count rate was $30 \%$ lower with the film on the lower side, indicating that despite tilting towards the detector, shadowing by grid bars was occurring in this orientation. Detector dead time was higher with the film uppermost (17-18\% vs $12-14 \%$ for the film on the lower side), reflecting the higher count rate due to an absence of shadowing. With the film uppermost, scattered electrons have a greater chance of interacting with the Mo grid below, compared with when the film is on the lower side of the specimen. Such interactions can produce greater intensities of high energy $\mathrm{x}$-rays (Mo $\mathrm{K}_{\alpha}$ $17.48 \mathrm{keV})$. This was apparent as a reduced $\mathrm{Ni} \mathrm{K}_{\alpha} / \mathrm{Mo} \mathrm{K}_{\alpha}$ ratio when the film was uppermost.

The greater interaction of high energy electrons scattered from the top mounted film onto the support grid, results in more intense Bremsstrahlung (Egerton and Cheng, 1994) leading a lower peak to background ratio - described by the Fiori number (Fiori and others, 1979). With the film uppermost this value was 4190 versus 4551 with the film lowermost. This value is the background 
subtracted counts in the $\mathrm{Ni}_{\alpha}$ peak divided by the counts in an adjacent equivalently-sized energy window $(10 \mathrm{eV})$ on the background. The $\mathrm{Mo} \mathrm{K}_{\alpha} / \mathrm{Mo}_{\alpha}$ ratio is a function of the nature of the stray radiation causing Mo excitation. The very low values observed here indicate that stray electrons, rather than hard $\mathrm{x}$-rays from the condenser aperture, are the primary source of Mo excitation. A hard $\mathrm{x}$-ray aperture to block such $\mathrm{x}$-rays was used throughout. Experience with the JEOL ARM instrument shows that the hard $\mathrm{x}$-ray aperture does result in minor improvements in eliminating unwanted system peaks. However, in most cases it is not required.

Low energy efficiency is a measure of the sensitivity of the detector to $\mathrm{O} K \mathrm{x}$-rays relative to those of $\mathrm{Ni} K_{\alpha}$. The high values seen here (unity is ideal) reflect the highly efficient design of solid state detectors and the high transparency of the atmospheric thin window. Tilting towards the detector results in an increased take-off angle - which reduces absorption within the film. The reduced sensitivity for the downward-facing film may in part be caused by partial shadowing by the grid. Also important is the fact that the NiO film is placed on top of a $25 \mathrm{~nm}$ carbon support film (on an Mo grid). With the film on the lower side of the specimen, $x$-rays must pass upwards through the carbon layer to reach the detector, resulting in some absorption of the low energy end of the spectrum.

Solid angle is a critical parameter for an EDS detector, and the development of very large solid angle detectors in recent years has resulted in a step function improvement in the sensitivity and throughput of analytical STEM. The measurement of probe current is an essential prerequisite in computing the solid angle (Egerton and Cheng, 1994). In the case of the JEOL detector the specimen orientation has a strong effect on solid angle (Table 3). With the film on the underside of the grid, the solid angle was reduced by $30 \%$. This is caused by shadowing of the detector by the grid. The average solid angle obtained with the film uppermost was $0.72+-0.03 \mathrm{sr}$. The largest solid angle obtained from a set of four measurements was $0.75 \mathrm{sr}$. The apparent discrepancy between the reported solid angle (1sr) and the measured value may be accounted for by the presence of silicon support grid immediately behind the atmospheric thin window of this detector. This grid provides mechanical support to enable the atmospheric thin window (ATW) to withstand atmospheric pressure. Typical transmission through such a grid might be $70 \%$ and so a measured solid angle of $0.7 \mathrm{sr}$ would then correspond to a geometric solid angle of $1 \mathrm{sr}$ (which only considers geometry and ignores the support grid).

\section{FEI Super-X Windowless Detector: Detecting Ice and Carbon}

One further very useful attribute of the NiOx specimen is that it enables the presence of ice and carbon on the detector to be monitored. Older generation SiLi detectors are cooled to liquid nitrogen temperature and are kept cold permanently. The ATW should act as a barrier to vacuum contaminants. However, hydrocarbons and water may still condense on the detector during its $10-$ 15 year life span, especially if pinholes in the window are present, or if the window is permeable. Contaminants may also build up on the vacuum side of the ATW. The presence of these contaminant layers reduces the light element sensitivity, and many vendors provide detector crystal warming routines to sublime away such films. The situation is different for most SDDs, in that they are Peltier cooled and so, typically, are not kept cold permanently. However, the Super- $X$ detector array is somewhat of a hybrid of these two situations. The array is thermally coupled to the column cold 
trap, which is cooled to liquid nitrogen temperature. The detectors are maintained at $-70{ }^{\circ} \mathrm{C}$ and so are considerably warmer than the surrounding cold trap. Since the detectors are windowless, there is the possibility of contaminants from the vacuum/specimen condensing onto the detector, necessitating a warm-up cycle. To evaluate this, the detector/anticontamination device was warmed to remove all contaminants, as per the manufacturer's recommendations. The vacuum system was left to stabilize in this condition over the weekend. Following cool down of the cold trap/detector array, NiOx spectra were obtained with the detector in the 'clean' condition. Thereafter, the system was kept cold and the microscope operated normally. Measurements of ice and carbonaceous deposits on the detector were made using the NiOx specimen over a period of two months. The presence of ice and carbonaceous species will reduce the sensitivity of the detector to Ni $\mathrm{L}_{\alpha}$ $(0.851 \mathrm{keV})$ relative to that for $\mathrm{Ni} \mathrm{K}_{\alpha}(7.477 \mathrm{keV}) \mathrm{x}$-rays. On the other hand, ice has little effect on the absorption of $\mathrm{O} \mathrm{K} x$-rays, since the $\mathrm{O} \mathrm{K}$ photon energy $(0.525 \mathrm{keV})$ lies below the absorption edge for oxygen ( $0.535 \mathrm{keV})$. However, carbonaceous layers will cause strong $\mathrm{O} \mathrm{K}$ absorption. A combination of $\mathrm{Ni}_{\alpha} / \mathrm{Ni} \mathrm{K} \mathrm{K}_{\alpha}$ and $\mathrm{OK} / \mathrm{Ni} \mathrm{K}_{\alpha}$ measurements for a clean and an iced detector, enable the thickness of such layers to be obtained (NiOx EDS reference specimen, 2015). The SuperX Monitor script (Mitchell, 2014c) allows data from a clean detector to be saved and when subsequent measurements of the detector are then carried out ice and carbon thickness are reported.

Fig. 5 shows the thickness of ice and hydrocarbons on the Super-X detector over a two month period. By definition the initial measurement is for the 'clean' detector immediately following coldtrap/detector cool-down. The rapid build-up of ice and carbon over the first seven days is not unexpected, since when the system is first cooled, the cold trap has not yet cleaned up the vacuum and the column vacuum contaminant levels are at their highest. The thickness of ice on the detector peaks earlier than that of the hydrocarbon layer, perhaps as a result of the higher vapour pressure/mobility of water. However, the thickness of both contaminant films then falls with time. Most likely this is the result of migration of contaminants from the detector array $\left(-70{ }^{\circ} \mathrm{C}\right)$ to the adjacent cold trap $\left(-196{ }^{\circ} \mathrm{C}\right)$, as a result of radiation-induced sublimation. As the cold trap progressively cleans the vacuum, the rate of sublimation of contaminants exceeds the rate of arrival, and the detector self cleans. Ice is completely gone from the detector by 60 days and only $10 \mathrm{~nm}$ of carbon remains. There appears to be merit in keeping the detector array cold for periods well beyond the recommended monthly warm-up cycles. For operational convenience warm-up cycles are initiated only during extended end of year vacation breaks.

\section{Conclusions}

Investigation of practical methods of measuring TEM/STEM probe currents have been explored. In the absence of picoammeter, a DMM is an inexpensive alternative, which can provide high accuracy, provided the internal resistance is known. The DMM can be used for measuring the voltage developed across the outputs of the drift tube of an EELS spectrometer. The drift tube is an effective Faraday Cup, and does not suffer the current losses inherent in measurements using a fluorescent screen. These arise from backscattered and secondary electron signals. The magnitude of these losses have been determined at various voltages by comparison with drift tube-determined currents. Direct correlation of fluorescent screen-derived current density with that measured at the drift tube, enables facile probe current measurement, and avoids the complexities of determining screen area 
and BSE and SE losses. Correlation of the drift-tube determined probe currents with intensities generated on two different CCD cameras have also been made to enable the conversion efficiency to be determined. This enables the calibration of images in terms of electrons. The efficiency with which the electron energy is converted into a CCD signal increases with decreasing accelerating voltage and is also scintillator-dependent. Calibrated probe currents enable the solid angle of the $x$ ray detector to be determined via the use of a $\mathrm{NiO}$ reference specimen. This specimen also permits a range of other measurements to be made. Some performance characteristics of large area EDS detectors are described. Many of the measurements described herein have been automated via a series of scripts for DigitalMicrograph, which are freely available from the internet. 
Acknowledgements

This research used the JEOL ARM200F microscope funded by the Australian Research Council (ARC) Linkage, Infrastructure, Equipment and Facilities (LIEF) grant LE120100104 located at the UOW Electron Microscopy Centre. 
References

Bai X-c, Fernandez IS, McMullan G, Scheres SH. 2013. Ribosome structures to near-atomic resolution from thirty thousand cryo-EM particles. Kühlbrandt W, editor.

Dubochet J, Adrian M, Chang J-J, Homo J-C, Lepault J, McDowall AW, Schultz P. 1988. Cryo-electron microscopy of vitrified specimens. Quarterly Reviews of Biophysics 21(02): 129-228.

Egerton RF, Cheng SC. 1994. Characterization of an analytical electron microscope with a NiO test specimen. Ultramicroscopy 55(1): 43-54.

Egerton RF, Li P, Malac M. 2004. Radiation damage in the TEM and SEM. Micron 35(6): 399-409.

Fiori CE, Swyt CR, Ellis JR. 1979. An introduction to analytical electron microscopy.

Gatan. 2015. http://www.gatan.com/products/tem-specimen-holders/analytical-holders.

Goldstein J, Newbury DE, Joy DC, Lyman CE, Echlin P, Lifshin E, Sawyer L, Michael JR. 2003. Scanning electron microscopy and x-ray microanalysis.

Jiang $\mathrm{N}$, Spence JCH. 2012. On the dose-rate threshold of beam damage in TEM. Ultramicroscopy 113(0): 77-82.

Mackovic M, Niekiel F, Wondraczek L, Spiecker E. 2014. Direct observation of electron-beam-induced densification and hardening of silica nanoballs by in situ transmission electron microscopy and finite element method simulations. Acta Mater. 79: 363-373.

Mitchell DRG. 2014a. http://www.dmscripting.com/ccd electron calibrator.html.

Mitchell DRG. 2014b. http://www.dmscripting.com/current converter.html.

Mitchell DRG. 2014c. http://www.dmscripting.com/super-x monitor.html.

NiOx EDS reference specimen TPi. 2015. http://www.tedpella.com/calibration html/TEM7.htm.

Sader K, Brown A, Brydson R, Bleloch A. 2010. Quantitative analysis of image contrast in phase contrast STEM for low dose imaging. Ultramicroscopy 110(10): 1324-1331.

Sousa AA, Leapman RD. 2007. Quantitative STEM mass measurement of biological macromolecules in a 300 kV TEM. J. Microsc. (Oxford, U. K.) 228(1): 25-33.

Trump JG, Van De Graaff RJ. 1947. The Insulation of High Voltages in Vacuum. Journal of Applied Physics 18(3): 327-332.

Williams DB, Carter CB. 2009. Transmission Electron Microscopy: A textbook for Materials Science.

Zaluzec NJ. 2014. Calculation and measurements of XEDS collection solid angle in TEM. Annual Conference on Microscopy and Microanalysis. Adelaide.

Zuo JM. 2000. Electron detection characteristics of a slow-scan CCD camera, imaging plates and film, and electron image restoration. Microscopy Research and Technique 49(3): 245-268. 
Table 1 Correction factors for current measurements using a fluorescent screen

\begin{tabular}{|l|c|c|}
\hline & $80 \mathrm{kV}$ & $200 \mathrm{kV}$ \\
\hline BSE/SE Loss Correction Factor & 1.52 & 1.18 \\
\hline
\end{tabular}

Table 2 CCD Conversion Efficiencies for two different cameras at 80 and 200kV

\begin{tabular}{|l|c|c|}
\hline \multicolumn{1}{|c|}{ Camera } & 80kV / counts per electron & 200kV / counts per electron \\
\hline Top Mounted Ultrascan (P+) & 9.9 & 4.8 \\
GIF Mounted Ultrascan (P) & 16.8 & 8.0 \\
\hline ( ) scintillator type
\end{tabular}

Table 3 Some JEOL Large Area EDS detector parameters obtained at 200kV (probe $=0.356 n A$ ).

\begin{tabular}{|l|l|l|l|l|l|l|l|l|}
\hline $\begin{array}{l}\text { Film } \\
\text { Position }\end{array}$ & $\begin{array}{l}\text { Count } \\
\text { Rate/cps }\end{array}$ & $\begin{array}{l}\text { Dead } \\
\text { Time/\% }\end{array}$ & $\begin{array}{l}\text { Mn } \mathrm{K}_{\alpha} \\
\text { Resolution } \\
\text { /eV }\end{array}$ & $\begin{array}{l}\mathrm{Ni} \mathrm{K}_{\alpha} \\
\text { Fiori }\end{array}$ & $\begin{array}{l}\mathrm{NiK}_{\alpha} / \\
\text { Mo K }_{\alpha}\end{array}$ & $\begin{array}{l}\text { Mo K }_{\alpha} / \\
\text { Mo } \mathrm{L}_{\alpha}\end{array}$ & $\begin{array}{l}\text { Low } \\
\text { Energy } \\
\text { Efficiency }\end{array}$ & $\begin{array}{l}\text { Solid } \\
\text { Angle / } \\
\text { sr }\end{array}$ \\
\hline $\begin{array}{l}\text { Upper } \\
\text { Side }\end{array}$ & 12100 & $17-18$ & $\begin{array}{l}138.6 \\
(0.3)\end{array}$ & $\begin{array}{l}4189 \\
(96)\end{array}$ & $\begin{array}{l}44.6 \\
(1.6)\end{array}$ & $\begin{array}{l}1.27 \\
(0.09)\end{array}$ & $\begin{array}{l}0.80 \\
(0.015)\end{array}$ & $\begin{array}{l}0.72 \\
(0.03)\end{array}$ \\
$\begin{array}{l}\text { Lower } \\
\text { Side }\end{array}$ & 9100 & $12-14$ & $\begin{array}{l}137.75 \\
(0.3)\end{array}$ & $\begin{array}{l}4551 \\
(122)\end{array}$ & $\begin{array}{l}46.1 \\
(5.1)\end{array}$ & $\begin{array}{l}1.34 \\
(0.06)\end{array}$ & $\begin{array}{l}0.63 \\
(0.01)\end{array}$ & $\begin{array}{l}0.51 \\
(0.06)\end{array}$ \\
\hline
\end{tabular}


Figure Legends

Fig. $1 \mathrm{FEl}$ : Comparison of the measured (drift tube) probe current with that indicated on the FluCam screen of the Osiris microscope.

Fig 2. Plot of Drift Tube current compared with that measured on the large fluorescent screen of the JEOL ARM (uncorrected for backscatter and secondary electron losses) at 200kV. The non-unity slopes $(200 \mathrm{kV}=1.18,80 \mathrm{kV}=1.52)$ reflect the incomplete current collection by the fluorescent screen.

Fig. 3 Relationship between drift tube measured current and the screen current density for the large and small fluorescent screens on the JEOL ARM at 200kV.

Fig. $4 \mathrm{X}$-ray count rate (corrected for thickness variation with tilt) as a function of specimen tilt (positive tilt is towards the detector) for the JEOL large area EDS detector on the ARM at 200kV.

Fig. 5 Ice and carbon thickness formed on the windowless FEI Super-X detector array following initial cool down. 


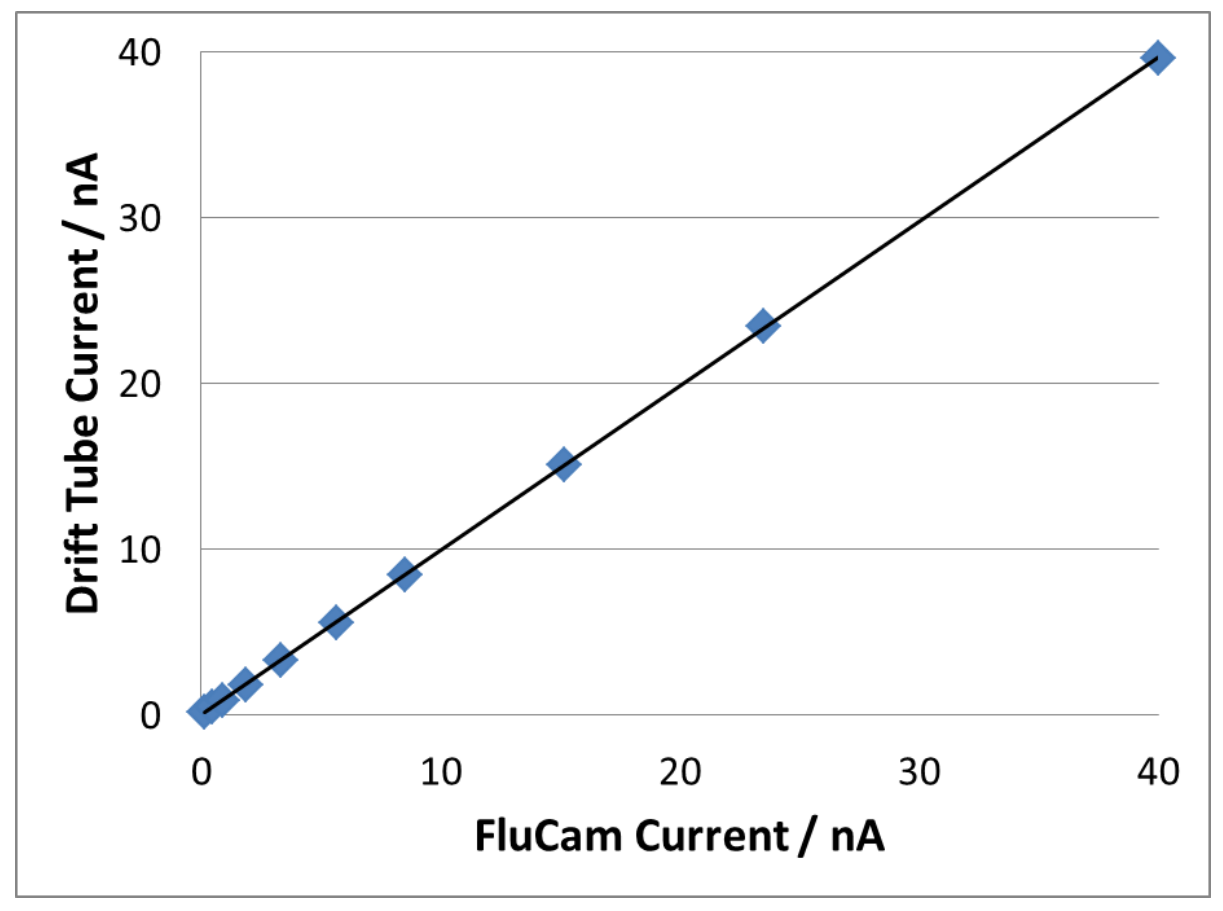

Fig. 1 FEl: Comparison of the measured (drift tube) probe current with that indicated on the FluCam screen of the Osiris microscope.

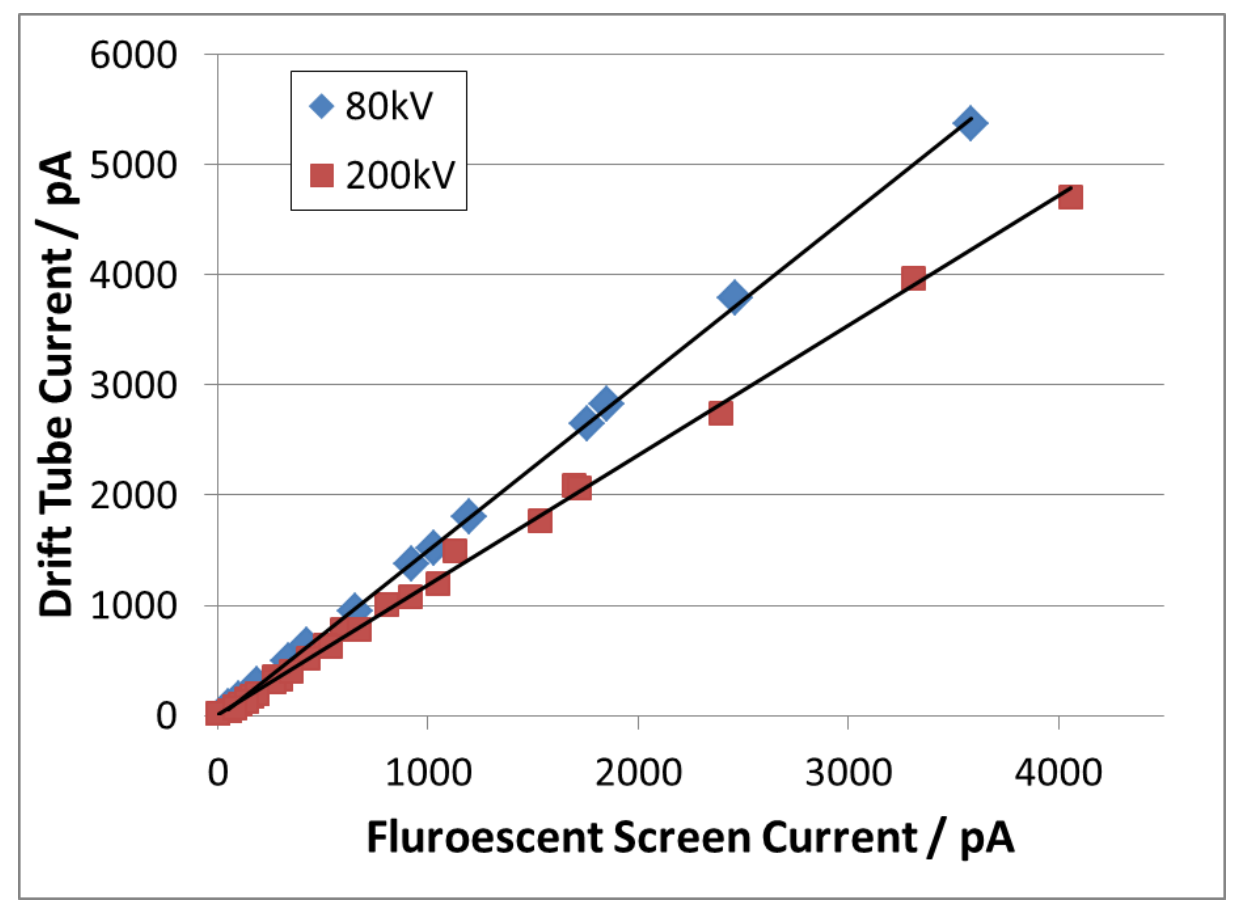

Fig 2. Plot of Drift Tube current compared that measured on the large fluorescent screen of the JEOL ARM (uncorrected for backscatter and secondary electron losses) at 200kV. The non-unity slopes $(200 \mathrm{kV}=1.18,80 \mathrm{k} V=1.52)$ reflect the incomplete current collection by the fluorescent screen. 


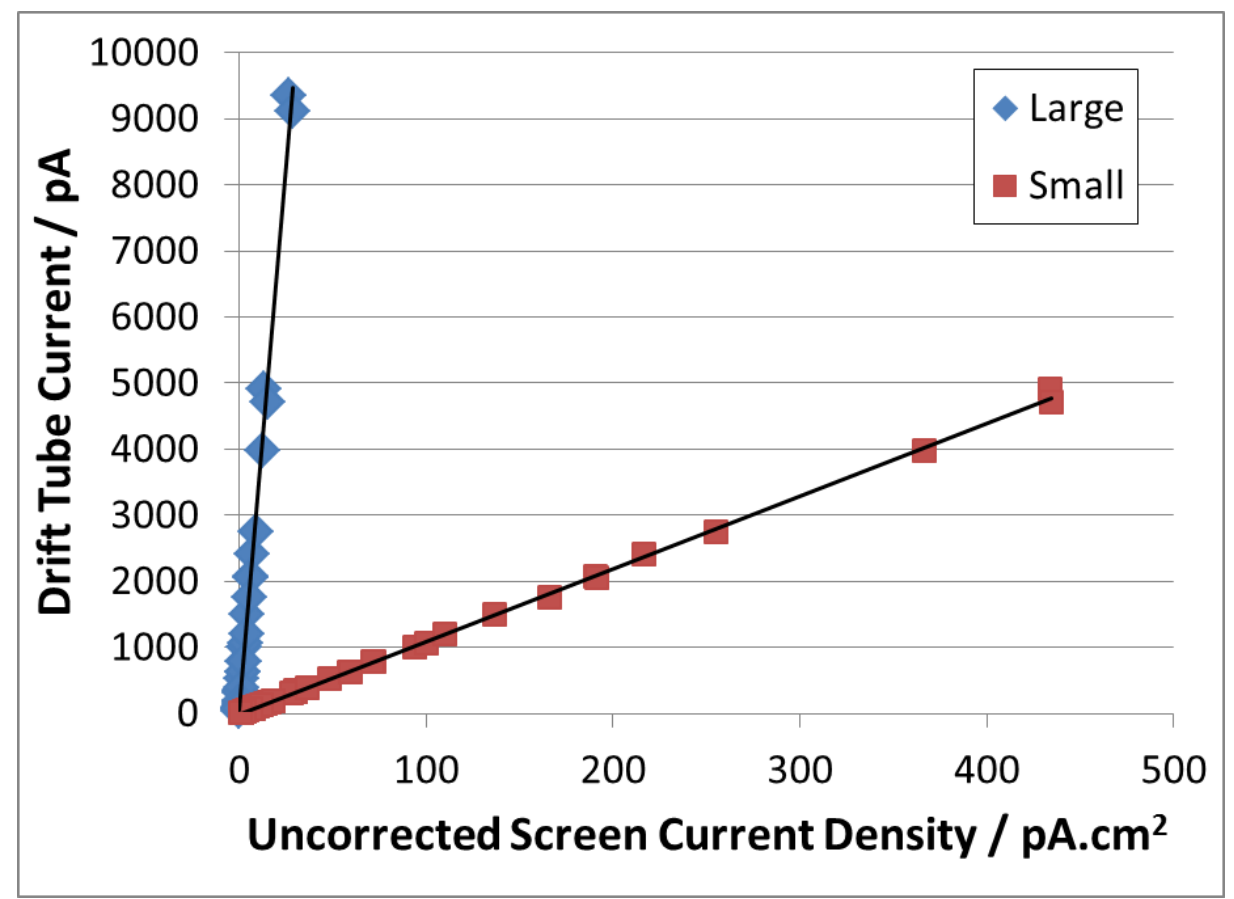

Fig. 3 Relationship between drift tube measured current and the screen current density for the large and small fluorescent screens on the JEOL ARM at 200kV.

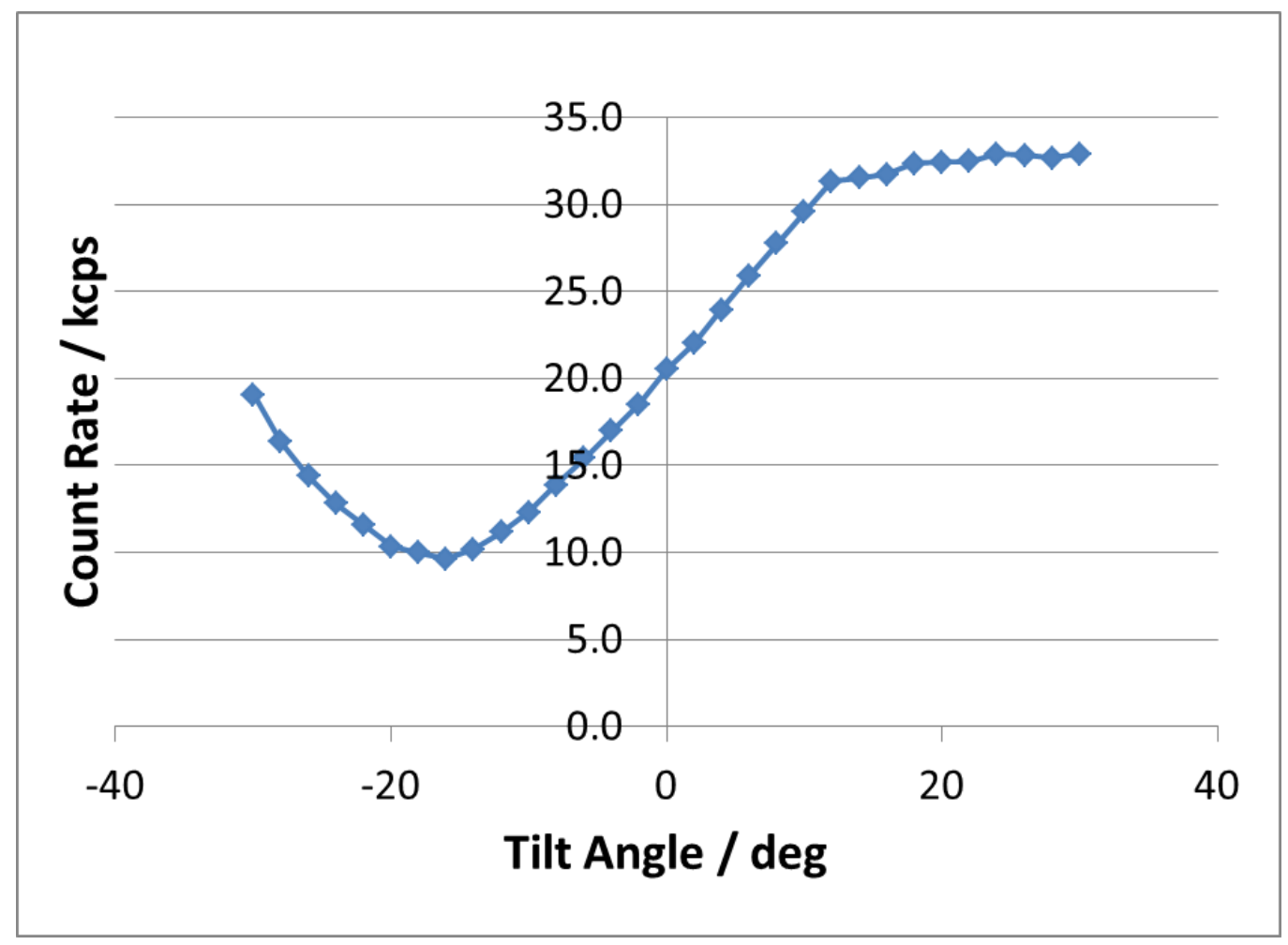

Fig. $4 \mathrm{X}$-ray count rate (corrected for thickness variation with tilt) as a function of specimen tilt (positive tilt is towards the detector) for the JEOL large area EDS detector. 


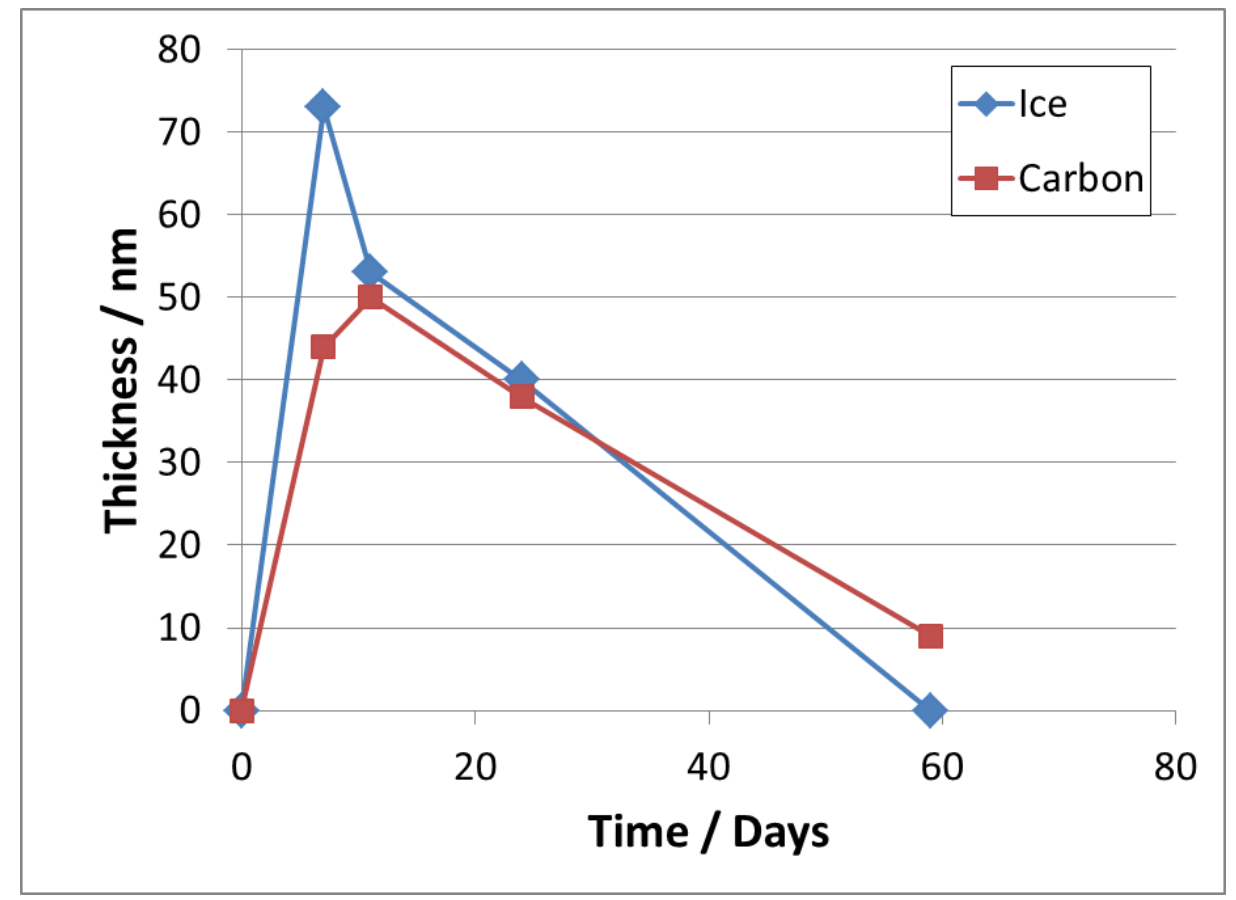

Fig. 5 Ice and carbon thickness formed on the windowless FEI Super-X detector array following initial cool down. 